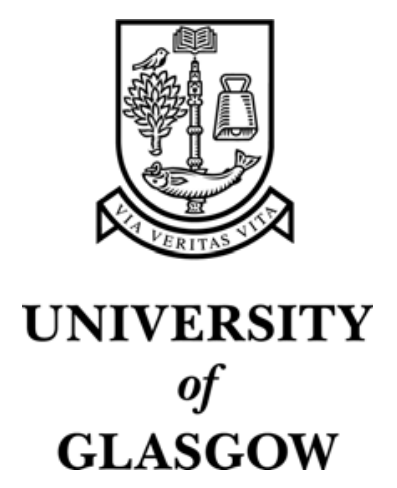

Ashrafi, G.H. and Brown, D.R. and Fife, K.H. and Campo, M.S. (2006) Down-regulation of MHC class I is a property common to papillomavirus E5 proteins. Virus Research 120(1-2):pp. 208-211.

http://eprints.gla.ac.uk/3088/ 
Virus Research, 120 (2006) 208-211

\title{
Down-regulation of MHC class I is a property common to papillomavirus E5 proteins
}

\author{
G.H. Ashrafi ${ }^{a}$, D.R. Brown ${ }^{\text {, }}$, K.H. Fife ${ }^{\mathrm{b}}$, M.S. Campo ${ }^{\mathrm{a}, *}$ \\ aivision of Pathological Sciences, Institute of Comparative Medicine, University of Glasgow, Garscube Estate, Glasgow G61 1QH, Scotland, UK ${ }^{\mathrm{b}}$ Department of Medicine, Indiana \\ University School of Medicine, Indianapolis, IN, USA \\ Received 18 November 2005; received in revised form 8 February 2006; accepted 8 February 2006 \\ Available online 15 June 2006
}

\begin{abstract}
The E5 protein family of papillomaviruses comprises small hydrophobic proteins which are associated with the cell endomembrane compartments. The functions of the E5 proteins, particularly those of HPV, are still far from clear. We have reported that the E5 proteins of BPV1, BPV-4, HPV-16 and HPV-6 down-regulate MHC class I, potentially helping the virus evade the host immune response. Others have described MHC class I down-regulation by HPV-2 E5. We report here that another E5 protein, HPV-83 E5, likewise down-regulates MHC class I and propose that interference with expression, assembly and/or transport of MHC class I is a common property of all E5 proteins evolved by the virus to circumvent host immunosurveillance and thus establish productive infection. (c) 2006 Elsevier B.V. All rights reserved.
\end{abstract}

Keywords: HPV-83; E5; MHC class I down-regulation; E5-heavy chain interaction

Viruses have to evade the innate and adaptive host immune response to establish infection and persist at least until new progeny virus is released. Many viruses down-regulate the major histocompatibility complex (MHC) class I (Piguet, 2005), thus avoiding lysis by cytotoxic T lymphocytes (CTL; effector cells capable of recognising and destroying transformed or infected cells).

Papillomaviruses (PV), small oncogenic viruses that infect mucosal and cutaneous epithelia, have also developed the ability to interfere with the expression and/or transport of MHC class I to the cell surface, thus potentially evading the host immune response (O’Brien and Campo, 2002) and the viral protein responsible for down-regulation of MHC class I is the E5 oncoprotein. This has been demonstrated for bovine papillomavirus type 4 (BPV-4) and BPV-1 E5, and human papillomavirus type 16 (HPV-16), HPV-6 and HPV-2 E5 (Ashrafi et al., 2002, 2005; Cartin and Alonso, 2003; Marchetti et al., 2002). The E5 proteins from different PV share a number of characteristics: they are small hydrophobic peptides located in the endomembrane compartments of the infected cell, interact with the $16 \mathrm{k}$ subunit $\mathrm{c}$ of the vacuolar $\mathrm{H}^{+}-$ ATPase, contribute directly or indirectly to the activation of growth factor receptors and, as pointed above, down-regulate MHC class I (Suprynowicz et al., 2005; Tsai and Chen, 2003). Despite these functional similarities, there is little amino acid homology between the various E5 proteins, their length varies from 42 amino acids in BPV-4 to 83 amino acids in HPV-16 to 91 amino acids in HPV-6; a number of HPV E5 proteins are close in length to BPV E5, these include the E5 proteins of HPV-2a and HPV-83.

HPV-83, previously called HPV IU (Brown et al., 1998), is a member of the A3 phylogenetic subgroup of genital papillomaviruses (Bernard, 2005; de Villiers et al., 2004). HPV-83 has been identified in approximately 1-5\% of clinical specimens from women with normal cytology and from 3 to 20\% of patients with cervical dysplasia (Brown et al., 2002; Giuliano et al., 2002;

Chaturvedi et al., 2005). It has also been shown to infect 3\% of college women who acquire new HPV infections each year (Ho et al., 1998). Interestingly, HPV-83 is one of the most common types identified in men and women with human immunodeficiency virus (HIV) infection (Broker et al., 2001; Chaturvedi et al., 2005; Kreuter et al., 2005). Although there is some uncertainty, the identification of HPV-83 in specimens from patients with cervical dysplasia and cancer suggests that the virus is probably not a low risk HPV type. Some histological features of the HPV-83-infected human 
foreskin implants grown in athymic mice also suggest that this virus is capable of causing dysplasia (Brown et al., 1998). The predicted E7 oncoprotein of HPV-83 has structural features similar to the high-risk HPV types. Three potential ORFs are present in the E5 region of the viral genome (Brown et al., 1999). The ORF most likely to be bona fide E5 ORF (called E5c in the initial publication) is postulated to encode a 46 amino acid long protein with structural features similar to BPV E5 (Brown et al., 1999). Nothing is known about the function of this protein.

As part of our investigation into the relationship between papillomavirus E5 proteins and the major histocompatibility complex (MHC) class I of the host, we decided to examine whether this putative HPV-83 E5 would down-regulate MHC class I expression like other E5 proteins (Ashrafi et al., 2002, 2005; Cartin and Alonso, 2003).

The E5c ORF of HPV-83 was amplified from full-length viral DNA using primers located at positions 4058 and 4278 and cloned into the pcDNA vector (Invitrogen, Carlsbad, CA) both in the sense (pc83E5s) and antisense (pc83E5a) orientation. Recombinant plasmids or empty vector (4 mg of each) were transfected into the immortalized human keratinocyte HaCaT cell line and clones were isolated, as described before (Ashrafi et al., 2005).

Clonal populations of cells were analysed for expression of both surface and total (surface plus intracellular) MHC class I by flow cytometry with W6/32, a monoclonal antibody (mAb) that recognises monomorphic conformational epitopes in the fully assembled MHC class I (Serotec, UK). Briefly, intact or permeabilised cells (0.5\% saponin in PBS for 30 min) were incubated with mAb W6/32 (1:100), and then with 1:100 dilution of anti-mouse IgG-FITC (Sigma, St. Louis, MO). The cells were then examined in a Beckman Coulter EPICS Elite analyzer equipped with an ion argon laser with $15 \mathrm{mV}$ of excitation at $488 \mathrm{~nm}$. Data were analyzed using Expo 2 software (see Ashrafi et al., 2005 for full protocol).

All clones of HaCaT keratinocytes harbouring HPV-83 E5 (HaCaT-83E5s) had much less surface MHC class I than either parental HaCaT cells or HaCaT cells harbouring pcDNA empty vector or the $83 \mathrm{E} 5$ antisense construct (Fig. 1). The extent of down-regulation of surface MHC class I by 83E5 was comparable to that brought about by HPV-16 E5 (16E5). However, in contrast to the situation in 16E5-expressing cells where total MHC class I was not affected, total MHC class I was also noticeably reduced by $83 \mathrm{E} 5$ (Fig. 1).

This finding was surprising. While the down-regulation of surface MHC class I by 83E5 was expected, as all the E5 proteins analysed so far induce it, the inhibition of total MHC class I was unforeseen as neither HPV-16 E5 nor HPV-6 E5a significantly affects its level (Ashrafi et al., 2005).

MHC class I is a ternary complex made up of heavy chain (HC), $\beta 2$ microglobulin $(\beta 2 \mathrm{M})$ and peptide, all of them essential for the formation and stability of the complex. Peptide is transported to MHC class I by TAP (transporter associated with peptide), a chaperon critical for the formation of the complex (Cresswell et al., 1999). The BPV E5 proteins prevent complex formation by inhibiting the expression of the HC (Marchetti et al., 2006).

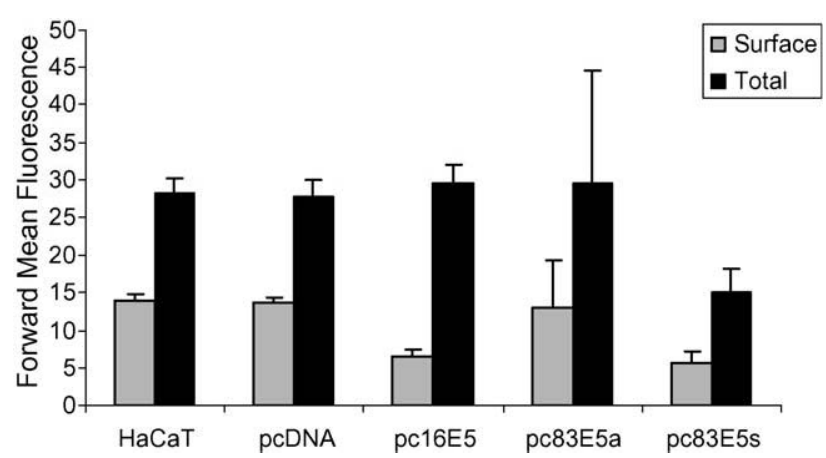

Fig. 1. HPV-83 E5 down-regulates MHC class I. Parental HaCaT cells, cells harboring empty vectors (pcDNA), pc16E5, pc83E5s (83E5 in sense orientation) or pc83E5a (83E5 in antisense orientation) were analyzed for expression of total and surface MHC class I by flow cytometry with $\mathrm{mAb}$ W6/32. The average mean fluorescence was calculated from the flow cytometric analyses of two duplicate measurements from at least two clones of each cell line. The background (the reading of cells stained with no primary antibody and only secondary antibody) was 0.4 in all cases. Standard deviation is shown.

To clarify whether this was the case also for 83E5, we analysed the expression of HC in the HaCaT-83E5 cells by immunoblotting with HC10, a mAb that recognises linear epitopes in the HC of HLA class I (1:50; a kind gift from Dr. Stephen Man, Cardiff University, Cardiff, UK). mAb AB-1 (1:20,000; Oncogene Research Products, Nottingham, UK) specific for actin was used as control (for full protocol, see Ashrafi et al., 2005). 
As shown in Fig. 2, the levels of HC were comparable between HaCaT-83E5 cells and the control cell lines, showing that 83E5

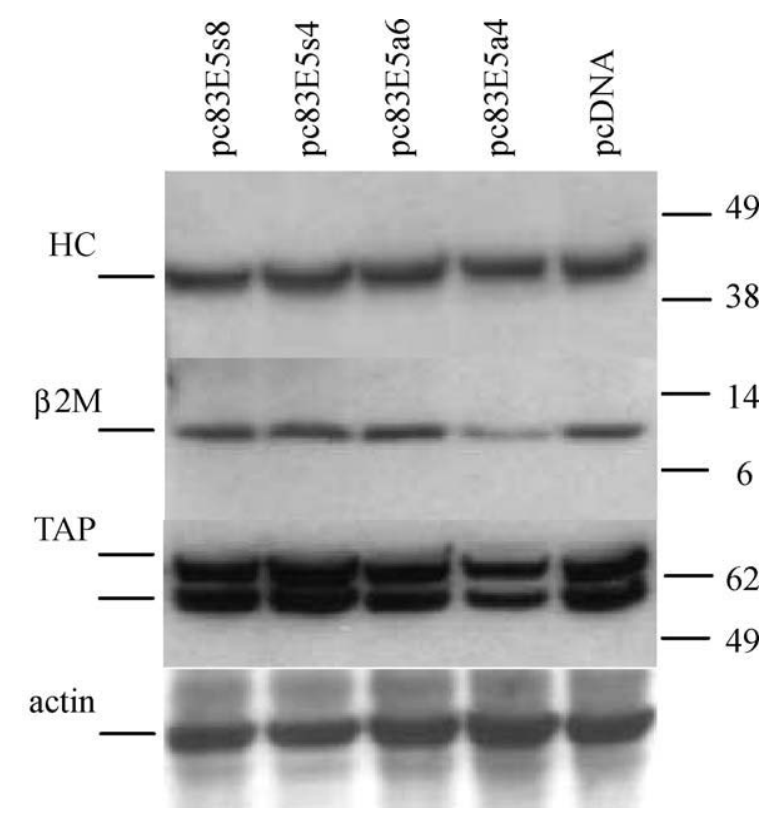

Fig. 2. HPV-83 E5 does not down-regulate expression of heavy chain, $\beta 2$ microglobulin or TAP. Equal amounts (10 $\mu$ g) of protein lysates from one line of HaCaT cells carrying empty vectors (pcDNA), two lines of HaCaT-83E5s (sense, pc83E5s4 and pc83E5s8), and two lines of HaCaT83E5a (antisense, pc83E5a4 and pc83E5a6) were analyzed by immunoblotting with mAb HC10 (anti-HC), mAb BBM.1 (anti- $\beta 2 \mathrm{M}$ ), polyclonal Ab 735-748 (anti-TAP) or mAb AB-1 (anti-actin). The two TAP bands correspond to TAP-1 and TAP-2. The numbers on the rights are molecular weight markers.

does not affect the expression of HC. Given that there are no differences in the levels of HC between HaCaT control and HaCaT-83E5 cells, but nevertheless the amount of total MHC class I is much less in the latter, we investigated whether the reduction in MHC class I was due to reduction in $\beta 2 \mathrm{M}$ or TAP, by immunoblotting with mAb BBM.1 (anti- $\beta 2 \mathrm{M}$; 1:500; CR, London, UK) or rabbit polyclonal antibody 735-748 (anti-TAP; 1:1000; Calbiochem, UK). Although there was somewhat less $\beta 2 \mathrm{M}$ and TAP in pc83E5a4 (antisense) cells than in the other cell lines, there were no differences in the levels of either $\beta 2 \mathrm{M}$ or TAP in pc83E5s8 or pc83E5s4 cells compared to HaCaT control cells (Fig. 2). Therefore, down-regulation of MHC class I by 83E5 does not appear to be due to inhibition of expression of either $\beta 2 \mathrm{M}$ or TAP, suggesting that 83E5 prevents the assembly of HC in the MHC complex.

We have shown that both BPV-4 E5 and HPV-16 E5 physically interact with the MHC class I HC in bovine and human cells respectively, and that the in vivo interaction is accurately reproduced in vitro (Marchetti et al., 2006; Ashrafi et al., 2006). We therefore investigated whether $83 \mathrm{E} 5$ can likewise bind MHC class I HC by co-immunoprecipitation of in vitro transcribed-translated ${ }^{35}$ S-labelled $83 E 5$ and the HC of HLA-A2. Briefly, in vitro transcription/translation reactions were performed using the $\mathrm{TNT}^{\circledR} \mathrm{T} 7$ Quick Coupled Transcription/Translation System (Promega, UK) in presence of Redivue 1-[ $[\mathrm{S}]$ methionine (Amersham Pharmacia Biotech, UK) following the manufacturer instructions and as described before (Marchetti et al., 2006). mAb HC10 precipitated both A2 HC and 83E5 (Fig. 3), indicating that 83E5 and $\mathrm{A} 2 \mathrm{HC}$ are present in a physical complex in vitro and, given that in vitro co-immunoprecipitation accurately reflects the interaction taking place within the cell (Marchetti et al., 2006), we suggest that, like the previously analysed E5 proteins, 83E5 can interact with HC also in HaCaT cells. Such interaction is likely to prevent the assembly of HC in MHC class I complex. 


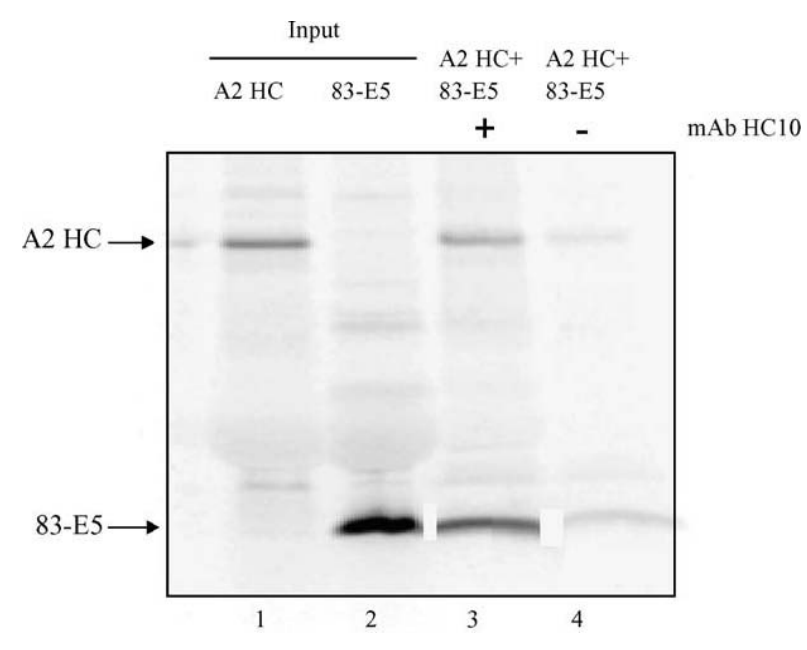

Fig. 3. HPV-83 E5 complexes with HLA-A2 heavy chain. A2 heavy chain (HC) and $83 E 5$ were transcribed/translated in vitro in the presence of ${ }^{35}$ S-methionine and precipitated with mAb HC10. Lane 1, A2 HC input; lane 2, 83E5 input; lane 3, A2 HC and 83E5 plus mAb HC10; lane 4, A2 HC and 83E5 minus mAb HC10.

It is interesting that the different E5 proteins achieve down-regulation of MHC class I in different ways and to a different extent (Table 1). BPV-4 E5 achieves an almost complete shut-off of MHC class I expression by both inhibiting transcription from the promoter of the heavy chain gene and inducing degradation of the heavy chain itself; furthermore any residual MHC class I complex is retained in the Golgi apparatus and prevented from reaching the cell surface (Ashrafi et al., 2002; Marchetti et al., 2002, 2006; Araibi et al., unpublished observations). HPV-16 E5 does not interfere with transcription or expression of the heavy chain or with complex assembly, but retains the complex in the Golgi apparatus and the amount of surface MHC class I is greatly reduced (Ashrafi et al., 2005). HPV-2 E5 down-regulates surface expression of MHC class I without decreasing expression of HC (Cartin and Alonso, 2003), but as the levels of total MHC class I were not measured, it is not known whether HPV-2 E5 impedes complex assembly. We suggest that HPV-83 E5 down-regulates MHC class I by preventing complex assembly.

Altogether, HPV-83 E5 has a more profound effect on MHC class I expression than HPV-16 E5. HPV83-E5 is more similar to BPV E5 both in length and in having only one hydrophobic putative trans-membrane helix, than to HPV16-E5 or HPV-6 E5a, which are approximately twice as long and have three hydrophobic domains (Bravo and Alonso, 2004). The E5 proteins of HPV have recently been classified in $\alpha, \beta, \gamma$ and $\delta$, depending on their physical characteristics such as position in the PV genome, length and hydrophobicity, and the classification correlates with the high or low risk nature of the HPVs (Bravo and Alonso, 2004). According to this classification, HPV-16 E5 falls in the $\alpha$ group, HPV-6 E5a in the $\gamma$ group, and HPV-2 E5 and HPV-83 E5 in the $\beta$ group. It is important to note that independently from their position in the genome, length or hydrophobicity, all the HPV E5 proteins ( $\alpha, \beta$ and $\gamma$ groups) and all the BPV E5 ( $\delta$ and $\xi$ genera) proteins tested so far down-regulate MHC class I to a greater or lesser extent. These observations strengthen our previous conclusions that the E5 proteins are important in the establishment of productive infection by helping the virus overcome the host immunosurveillance mechanisms through the down-regulation of MHC class I (Araibi et al., 2004; Ashrafi et al., 2002). The results also support the hypothesis that the HPV83 ORF analysed is the authentic E5 ORF as originally proposed (Brown et al., 1999).

The mechanisms employed by E5 to down-regulate MHC class I are still being elucidated but comprise at least two steps. One is the E5-induced inhibition of the acidification of the Golgi apparatus (Schapiro et al., 2000), as a similar down-regulation of surface MHC class I is seen in control cells treated with ionophores, which dissipate the $\mathrm{pH}$ gradient of the Golgi apparatus (Marchetti et al., 2002). The other is a direct interaction between E5 and the HC. BPV-4, BPV-1 and HPV-16 E5 proteins all interact with the HC (Marchetti et al., 2006; Ashrafi et al., 2006). Interaction between HPV83 E5 and HC may be the reason for lack of complex assembly. While these points remain to be established, downregulation of MHC class I looks increasingly an important common function of the E5 proteins. 
Table 1. Effects of E5 protein expression on MHC class I biogenesis.

\begin{tabular}{|l|c|c|c|c|c|}
\hline Inhibition of & $\begin{array}{c}\text { HC gene } \\
\text { transcription }\end{array}$ & HC stability & $\begin{array}{c}\text { Complex } \\
\text { assembly }\end{array}$ & $\begin{array}{c}\text { Complex } \\
\text { transport }\end{array}$ & Reference \\
\hline HPV-83 E5 & no & no & yes & yes & this paper \\
\hline BPV-4 E5 & yes & yes & nd & yes & $\begin{array}{c}\text { Ashrafi et al., } \\
\text { 2002; Marchetti } \\
\text { et al., 2002, 2005 }\end{array}$ \\
\hline BPV-1 E5 & yes & yes & nd & yes & $\begin{array}{c}\text { Ashrafi et al., } \\
\text { 2002; Marchetti } \\
\text { et al., 2002 }\end{array}$ \\
\hline HPV-16 E5 & no & no & no & yes & $\begin{array}{c}\text { Ashrafi et al., } \\
2005\end{array}$ \\
\hline HPV-6 E5 & nd & nd & no & yes & $\begin{array}{c}\text { Ashrafi et al., } \\
\text { 2005 }\end{array}$ \\
\hline HPV-2 E5 & nd & no & nd & yes & $\begin{array}{c}\text { Cartin and } \\
\text { Alonso, 2003 }\end{array}$ \\
\hline
\end{tabular}

nd: not done.

\section{Acknowledgements}

This work was partly financed by the Medical Research Council of Great Britain. M.S.C. is a Fellow of Cancer Research UK. 


\section{References}

Araibi, E.H., Marchetti, B., Ashrafi, G.H., Campo, M.S., 2004. Down-regulation of MHC class I in bovine papillomas. J. Gen. Virol. 85, 2809-2814.

Ashrafi, G.H., Haghshenas, M.R., Marchetti, B., O’Brien, P.M., Campo, M.S., 2005. The E5 protein of human papillomavirus type 16 selectively down-regulates surface HLA class. Int. J. Cancer 113, 276-283.

Ashrafi, G.H., Tsirimonaki, E., Marchetti, B., O’Brien, P.M., Sibbet, G.J., Andrew, L., Campo, M.S., 2002. Downregulation of MHC class I by bovine papillomavirus E5 oncoproteins. Oncogene 21, 248-259.

Ashrafi, G.H., Haghshenas, M., Marchetti, B., Campo, M.S., 2006. E5 protein of Human Papillomavirus 16 downregulates HLA class I and interacts with the heavy chain via its first hydrophobic domain. International Journal of Cancer, 119, 2105-2112.

Bernard, H.U., 2005. The clinical importance of the nomenclature, evolution and taxonomy of human papillomaviruses. J. Clin. Virol. 32 (Suppl. 1), S1-S6.

Bravo, I.G., Alonso, A., 2004. Mucosal human papillomaviruses encode four different E5 proteins whose chemistry and phylogeny correlate with malignant or benign growth. J. Virol. 78, 13613-13626.

Broker, T.R., Jin, G., Croom-Rivers, A., Bragg, S.M., Richardson, M., Chow, L.T., Vermund, S.H., Alvarez, R.D., Pappas, P.G., Squires, K.E., Hoesley, C.J., 2001. Viral latency-the papillomavirus model. Dev. Biol. (Basel) 106, 443-451.

Brown, D., Legge, D., Qadadri, B., 2002. Distribution of human papillomavirus types in cervicovaginal washings from women evaluated in a sexually transmitted diseases clinic. Sex. Transm. Dis. 29, 763-768.

Brown, D.R., McClowry, T.L., Bryan, J.T., Stoler, M., Schroeder-Diedrich, J.M., Fife, K.H., 1998. A human papillomavirus related to human papillomavirus MM7/LVX82 produces distinct histological abnormalities in human foreskin implants grown as athymic mouse xenografts. Virology 249, 150-159.

Brown, D.R., McClowry, T.L., Woods, K., Fife, K.H., 1999. Nucleotide sequence and characterization of human papillomavirus type 83, a novel genital papillomavirus. Virology 260, 165-172.

Cartin, W., Alonso, A., 2003. The human papillomavirus HPV2a E5 protein localizes to the Golgi apparatus and modulates signal transduction. Virology 314, 572-579.

Chaturvedi, A.K., Dumestre, J., Gaffga, A.M., Mire, K.M., Clark, R.A., Braly, P.S., Dunlap, K., Beckel, T.E., Hammons, A.F., Kissinger, P.J., Hagensee, M.E., 2005. Prevalence of human papillomavirus genotypes in women from three clinical settings. J. Med. Virol. 75, 105-113.

Cresswell, P., Bangia, N., Dick, T., Dietrich, G., 1999. The nature of the MHC class I peptide loading complex. Immunol. Rev. 172, 21-28.

de Villiers, E.M., Fauquet, C., Broker, T.R., Bernard, H.U., zur Hausen, H., 2004. Classification of papillomaviruses. Virology 324, 17-27.

Giuliano, A.R., Harris, R., Sedjo, R.L., Baldwin, S., Roe, D., Papenfuss, M.R., Abrahamsen, M., Inserra, P., Olvera, S., Hatch, K., 2002. Incidence, prevalence, and clearance of type-specific human papillomavirus infections: the young women's health study. J. Infect. Dis. 186, 462-469.

Ho, G.Y.F., Bierman, R., Beardsley, L., Chang, C.J., Burk, R.D., 1998. Natural history of cervicovaginal papillomavirus infection in young women. N. Engl. J. Med. 338, 423-428.

Kreuter, A., Brockmeyer, N.H., Hochdorfer, B., Weissenborn, S.J., Stucker, M., Swoboda, J., Altmeyer, P., Pfister, H., Wieland, U., 2005. Clinical spectrum and virologic characteristics of anal intraepithelial neoplasia in HIV infection. J. Am. Acad. Dermatol. 52, 603-608.

Marchetti, B., Ashrafi, G.H., Tsirimonaki, E., O’Brien, P.M.a., Campo, M.S., 2002. The papillomavirus oncoprotein E5 retains the major histocompatibility class I in the Golgi apparatus and prevents its transport to the cell surface. Oncogene 21, 7808-7816.

Marchetti, B., Ashrafi, G.H., Araibi, E.H., Ellis, S.A., Campo, M.S., 2006. The E5 protein of BPV-4 interacts with the heavy chain of MHC class I and irreversibly retains the MHC complex in the Golgi apparatus. Oncogene 25, 22542263.

O’Brien, P.M., Campo, M.S., 2002. Evasion of host immunity directed by papillomavirus-encoded proteins. Virus Res. 
$1-2,103-118$.

Piguet, V., 2005. Receptor modulation in viral replication: HIV, HSV, HHV-8 and HPV: same goal, different techniques to interfere with MHC-I antigen presentation. Curr. Top. Microbiol. Immunol. 285, 199-217.

Schapiro, F., Sparkowski, J., Adduci, A., Suprynowicz, F., Schlegel, R., Grin-stein, S., 2000. Golgi alkalinization by the papillomavirus E5 oncoprotein. J. Cell Biol. 148, 305-315.

Suprynowicz, F.A., Campo, M.S., Schlegel, R., 2005. Biological activities of papillomavirus E5 proteins. In: Campo, M.S. (Ed.), Papillomavirus Research: from Natural History to Vaccines and Beyond. Caister Academic Press, pp. 97113.

Tsai, T.C., Chen, S.L., 2003. The biochemical and biological functions of human papillomavirus type 16 E5 protein. Arch. Virol. 148, 1445-1453. 